\title{
UGT1A1*6, UGT1A7*3 and UGT1A9*1b polymorphisms are predictive markers for severe toxicity in patients with metastatic gastrointestinal cancer treated with irinotecan-based regimens
}

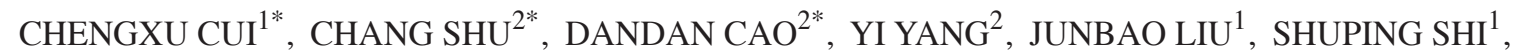 \\ ZHUJUN SHAO ${ }^{1}$, NAN WANG $^{1}$, TING YANG ${ }^{1}$, HAO LIANG $^{2}$, SHANSHAN ZOU $^{2}$ and SONGNIAN HU ${ }^{2}$ \\ ${ }^{1}$ Department of Medical Oncology, Cancer Institute and Hospital, Chinese Academy of Medical Sciences, Beijing 100021; \\ ${ }^{2}$ CAS Key Laboratory of Genome Sciences and Information, Beijing Institute of Genomics, \\ Chinese Academy of Sciences, Beijing 100101, P.R. China
}

Received March 30, 2015; Accepted August 3, 2016

DOI: $10.3892 / 01.2016 .5130$

\begin{abstract}
Irinotecan-induced severe neutropenia and diarrhea, which remain unpredictable, has restrained the dose and clinical efficiency of irinotecan administration. In the present study, a total of 70 irinotecan-treated patients with histologically confirmed metastatic gastrointestinal cancer were enrolled. Despite genotyping well-reported alleles, direct sequencing was specifically adopted to avoid ethnic heterogeneity and to identify novel variations. The promoter (-1000 bp) and exon 1 regions of UDP glucuronosyltransferase family 1 member A complex locus (UGT1A1) gene family members UGT1A1, UGT1A7 and UGT1A9 were sequenced, and comprehensive analysis of their genetic polymorphisms was performed to determine the association between inherited genetic variations and irinotecan-induced toxicity. A total of 23 different genetic variants were detected in the present study, including 2 novel polymorphisms. The results of the present study revealed that UGT1A $1^{*} 6$ and UGT1A7*3 are risk factors for irinotecan-induced severe neutropenia, and UGT1A9*1b
\end{abstract}

Correspondence to: Dr Chengxu Cui, Department of Medical Oncology, Cancer Institute and Hospital, Chinese Academy of Medical Sciences, 17 South Panjiayuan Lane, Beijing 100021, P.R. China

E-mail: cuichengxu@csco.org.cn

Dr Songnian $\mathrm{Hu}$, CAS Key Laboratory of Genome Sciences and Information, Beijing Institute of Genomics, Chinese Academy of Sciences, Building 104, 1 Beichen West Road, Beijing 100101, P.R. China

E-mail: husn@big.ac.cn

${ }^{*}$ Contributed equally

Key words: irinotecan, UGT1A, polymorphisms, neutropenia, diarrhea is associated with severe diarrhea. These results may provide biomarkers for the selection of the optimal chemotherapy for Chinese patients with metastatic gastrointestinal cancer.

\section{Introduction}

Irinotecan, also termed Camptosar and CPT-11, is a type of semi-artificial camptothecin compound that is one of the effective cytotoxic antineoplastic agents used to treat colorectal cancer (1). Irinotecan can be activated to SN-38 subsequent to hydrolysis of carboxylesterase in vivo, and derived into an inhibitor of topoisomerase I, which can synchronously be inactivated into SN-38G by UDP glucuronosyltransferase family 1 member A complex locus (UGT1A) (2). Thus, irinotecan interrupts the replication and transcription of DNA by preventing the relegation of single-stranded DNA and causes the death of tumor cells following a series of biotransformations. Irinotecan received authorization from the US Food and Drug Administration (FDA) in 1998, and has been approved as the primary therapy of metastatic adult colorectal cancer, combined with 5-fluorouracil (5-FU) and leucovorin (LV) (3). However, severe neutropenia and diarrhea, the primary toxicities of irinotecan, have restrained the dose and clinical efficiency of irinotecan (4). It has been reported that the curative effect and negative toxicity are each dependent on ethnicity (5-7).

The UGT1A gene family has 13 members in Homo sapiens, and is located on Chr2 (2q37). Each member is composed of a unique exon 1 , followed by four common exons (exons 2-5). Therefore, each functional protein of the UGT1A family has a specific $\mathrm{N}$-terminus, which is determined by the first exon sequence, to recognize the corresponding substrate. In addition, the last four mutual exons provide the identical $\mathrm{C}$ termini, which consists of 245 amino acids and guarantee the combination with uridine diphosphate glucuronic acid (8). UGT1A1, UGT1A7 and UGT1A9 have been shown to be the major effective factors during the inactivation of SN-38 $(9,10)$, and genetic polymorphism in the UGT1A family has also been 
shown to affect the variability of irinotecan toxicity during clinical application $(11,12)$.

Considering the complex mechanism involved in the association between the UGT1A family and toxicity of irinotecan, the FDA has instructed the manufacturer of irinotecan to revise the label and add the caution of relative toxicity and dose titration in patients with the UGT1A1*28 polymorphism, as well as the advice for patients to undergo allelic detection (13). For patients from the Asian population, UGT1A $1{ }^{*} 6$ is considered to be another potential risk factor that is responsible for the toxicity among the Asian population in addition to the UGT1A1 ${ }^{*} 28$ allele $(11,12,14)$. The Japanese Pharmaceuticals and Medical Devices Agency package insert for irinotecan states that individuals who are homozygous for the UGT1A $1 * 6$ or UCT1A $1 * 28$ alleles, or heterozygous with the UGT1A $1{ }^{*} 6 /{ }^{*} 28$ genotype, are at an increased risk of severe adverse events, particularly neutropenia (15). The impact of UGT1A7 polymorphism on irinotecan-induced toxicity has also been well-studied (16-18). In addition, UGT1A9*22 has been reported to be associated with the toxic potency of irinotecan (18-20). Considering the ethnicity-specific distribution of genetic polymorphisms, the predictive markers for severe toxicity in Chinese patients treated with irinotecan-based regimens have not been affirmatively reported by studies yet.

In the present study, direct sequencing was adopted to avoid ethnic heterogeneity and to identify novel variations. The first exon regions of UGT1A1, UGT1A7 and UGT1A9 were sequenced, and comprehensive analysis of genetic polymorphisms was performed to determine the association between inherited genetic variations and irinotecan-induced toxicity. The major aim of the current study was to identify toxicity predictable markers of Chinese metastatic gastrointestinal cancer patients treated with irinotecan-based regimens.

\section{Patients and methods}

Patients. In total, 70 patients with histologically-confirmed metastatic gastrointestinal cancer were enrolled from the Cancer Institute and Hospital, Chinese Academy of Medical Sciences (Beijing, China) between January 2012 and December 2014. All patients were treated with the irinotecan-based folinic acid, fluorouracil (5-FU) and irinotecan (FOLFIRI) or capecitabine and irinotecan (XELIRI) regimens. The study protocol was approved by the Ethics Committee of Beijing Chao-Yang Sanhuan Cancer Hospital (Beijing, China), which is affiliated with the Cancer Institute and Hospital of the Chinese Academy of Medical Sciences. Written informed consent was obtained from all patients prior to entering the study.

Eligibility criteria. All patients were diagnosed with histologically-confirmed gastrointestinal cancer and were undergoing their first irinotecan-based chemotherapy. The patients were aged $>18$ years and had an Eastern Cooperative Oncology Group (ECOG) performance status (PS) of 0-2 and a life expectancy of $\geq 3$ months.

Chemotherapy. The patients were treated with one of the following FOLFIRI-based regimens until progressive disease (PD), unacceptable toxicity, patient refusal or a medical decision to discontinue treatment. Patients treated with the FOLFIRI regimen received $180 \mathrm{mg} / \mathrm{m}^{2}$ irinotecan intravenously (IV), $400 \mathrm{mg} / \mathrm{m}^{2} \mathrm{LV}$ IV and $400 \mathrm{mg} / \mathrm{m}^{2} 5-\mathrm{FU}$ bolus, followed by $2,400 \mathrm{mg} / \mathrm{m}^{2} 5-\mathrm{FU}$ IV, all administered on day 1 , every 2 weeks. Patients treated with the XELIRI regimen received $120 \mathrm{mg} / \mathrm{m}^{2}$ irinotecan IV on days 1 and 8 , and $800 \mathrm{mg} / \mathrm{m}^{2}$ oral capecitabine twice per day on days 1-14 every 3 weeks.

Genotyping and genetic analysis. Peripheral venous blood samples were obtained from 70 patients with metastatic gastrointestinal cancer, who were treated with irinotecan-based regimens (37 with FOLFIRI and 33 with XELIRI) for isolation of genomic DNA. Genomic DNA was isolated using the QIAamp DNA blood mini kit (Qiagen, Inc., Valencia, CA, USA). The promoter (-1000 bp) and exon 1 regions of UGT1A1, UGT1A7 and UGT1A9 were resequenced to screen for single nucleotide polymorphisms (SNPs), using the DYEnamic ET terminator cycle sequencing kit (GE Healthcare, Chalfont St. Giles, UK) on the ABI Prism 3730xl DNA analyzer (Applied Biosystems; Thermo Fisher Scientific, Inc., Waltham, MA, USA). Polymerase chain reaction (PCR) was performed in a final volume of $15 \mu \mathrm{l}$, containing $30 \mathrm{ng}$ genomic DNA, 1*PCR Takara buffer $\left(\mathrm{Mg}^{2+}\right.$ plus; Takara Biotechnology Co., Ltd., Dalian, China), 2.5 pmol of each primer, 25 pmol deoxynucleotide triphosphates and $1 \mathrm{U}$ of Taq DNA polymerase (Takara Biotechnology Co., Ltd.). Following pre-denaturation at $93^{\circ} \mathrm{C}$ for $3 \mathrm{~min}$, amplification was performed under the following conditions for 32 cycles: Denaturation at $95^{\circ} \mathrm{C}$ for $30 \mathrm{sec}$; annealing at $58^{\circ} \mathrm{C}$ for $40 \mathrm{sec}$; and extension at $72^{\circ} \mathrm{C}$ for $2 \mathrm{~min}$. The primer sequences used are shown in Table I (21).

Base calling, quality assessment and polymorphism determination from DNA sequencing were analyzed using Phred, Phrap, Consed (http://www.phrap.org/phredphrapconsed. html) and PolyPhred (http://droog.gs.washington.edu/polyphred/). Haploview 4.2 (Broad Institute of MIT and Harvard, Cambridge, MA, USA (22) was used to estimate allele frequencies, test the Hardy-Weinberg equilibrium, measure pairwise linkage disequilibrium (LD), establish haplotypes, estimate haplotype frequency, and analyze the association between the haplotypes and toxicity. LD blocks were defined by the 'solid spine of LD' algorithm, and LD structure was exhibited using GOLD heatmap color scheme $(23,24)$. The allele designations were defined according to the UGT Allele Nomenclature Committee (25).

Statistical analysis. Patients underwent baseline evaluations, including physical examination, complete medical history, ECOG PS, complete blood count, and hepatic and renal function tests. Toxicity evaluations were performed according to National Cancer Institute Common Terminology Criteria for Adverse Events version 3.0 criteria. Associations between the clinical features and toxicity were analyzed by two-tailed Fisher's exact test or Student's t-test. In addition, associations between the alleles or genotypes and toxicity were analyzed by two-tailed Fisher's exact test. $\mathrm{P}<0.05$ was considered to indicate a statistically significant difference, as calculated by PLINK v1.07 (http://pngu.mgh.harvard.edu/ purcell/plink) (26). Due to the exploratory nature of the present study, no adjustments were made for multiple comparisons. 
Table I. Primer sequences.

\begin{tabular}{lll}
\hline Genes & Primer ID & \\
\hline UGT1A1 & P1_E1Amer sequences \\
& U1_E1AR & TCGTCCTTCTTCCTCTCTGG \\
& U1_E1BF & GCAGTGCATGCAAGAAGAAT \\
& U1_E1BR & TGTCTGGCTGTTCCCACTTA \\
UGT1A7 & U1_PF & CCAGAAGATGATGCCAAAGA \\
& U1_PR & GGTCATTCTCTACCCCAGCA \\
& U7_E1AF & AAAGCTGTCAGTCCACAAAGG \\
& U7_E1AR & AAACTCATATTGCAGCACAGG \\
UGT1A9 & U7_E1BF & AAGTCAAAAATACCATTGGATGAA \\
& U7_E1BR & GGAAGATCACTGAATTGCACAG \\
& U7_PR & TTCCTCTGGGGGTAGTGTAGAA \\
& U9_E1AF & TCTTTCCGTCGAACATGAGA \\
& U9_E1AR & CACATTCACTGCCAATGATTTA \\
& U9_E1BF & CCAAGGCAAAGACCATAAGCTA \\
& U9_E1BR & CAAACTCCTGCAATTTGAAAAA \\
& U9_PR & CATATACCCTGGAGGATCTGGA \\
& CTGACGAGTACACGCATTGG & CCTCTGACCTCAAGGAGTGC \\
& CAATGATTTACCCAAAAGAACAAG
\end{tabular}

F, forward; R, reverse; UGT1A, UDP glucuronosyltransferase family 1 member A complex locus.

\section{Results}

In total, 70 patients with histologically-confirmed metastatic gastrointestinal cancer, consisting of 37 patients with metastatic colorectal cancer and 33 patients with metastatic gastric cancer, were enrolled in the present study. The rates of grade 3-4 toxicity were as follows: Neutropenia, $18.57 \%(n=13)$; and diarrhea, $10 \%(n=7)$. These results are in accordance with a previous study of Asian patients (6). The clinical features of patients and the incidence of severe toxicity of the irinotecan-based regimens FOLFIRI and XELIRI are summarized in Table II.

A total of 23 different genetic variants were detected in the present study, all of the variants were consistent with the Hardy-Weinberg equilibrium $(\mathrm{P}>0.05)$. Genetic variations in the UGT1A1, UGT1A7 and UGT1A9 genes were compared with the genomic reference sequence AF039138.1. The amino acids were located in the reference sequences NP_000454.1 (UGT1A1), NP_061950.2 (UGTIA7) and NP_066307.1 (UGTIA9). Of these variants, 2 novel polymorphisms were identified: M06 and M09, which are located in the promoter regions of UGT1A9 and UGT1A7, respectively. UGT1A1*6, which is found only in Asian populations, had the highest frequency [minor allele frequency (MAF), 22.5\%] of the SNPs in UGT1A1 in the present study. The frequency of the UGT1A1*28 SNP(MAF,9.3\%) was comparable to the frequency previously found in the Asian population (MAF, 7.0\%), and was much lower compared with the frequency in Caucasian patients (MAF, 31.6\%), as previously reported $(11,20)$. Other known UGT1A alleles, UGT1A1*80, UGT1A1*81, UGT1A7*2, UGT1A7*3 and UGT1A9*1b (UGT1A9*22), were found concurrently. Table III lists the summary of identified polymorphisms in UGT1A1, UGT1A7 and UGT1A9.
Table II. Clinical features of 70 patients with histologically-confirmed metastatic gastrointestinal cancer.

\begin{tabular}{lc}
\hline Features & Value, $\mathrm{n}(\%)$ \\
\hline Age, years & \\
Median & 54 \\
Range & $25-77$ \\
Gender & \\
Male & $48(68.57)$ \\
Female & $22(31.43)$ \\
Tumor location & \\
Colon & $37(52.86)$ \\
Stomach & $33(47.14)$ \\
ECOG PS & \\
0 & $19(27.14)$ \\
1 & $46(65.71)$ \\
2 & $5(7.14)$ \\
TNM stage IV & $70(100.00)$ \\
Regimen & \\
FOLFIRI & $37(52.86)$ \\
XELIRI & $33(47.14)$ \\
Neutropenia grade 3-4 & $13(18.57)$ \\
Diarrhea grade 3-4 & $7(10.00)$ \\
\hline
\end{tabular}

ECOG PS, Eastern Cooperative Oncology Group performance status; TNM, tumor-node-metastasis classification of malignant tumours; FOLFIRI, folinic acid, fluorouracil and irinotecan; XELIRI, capecitabine and irinotecan. 
Table III. Summary of identified polymorphisms in UGT1A1, UGT1A7 and UGT1A9.

\begin{tabular}{|c|c|c|c|c|c|c|c|}
\hline ID & Location & db SNP ID & Change & Allele & Function & MAF & H-Wpval \\
\hline M01 & UGT1A9 promoter & rs3806598 & $\mathrm{A}>\mathrm{C}$ & - & - & 0.243 & 0.747 \\
\hline M02 & UGT1A9 promoter & rs45440791 & $\mathrm{T}>\mathrm{C}$ & - & - & 0.007 & 1.000 \\
\hline M03 & UGT1A9 promoter & rs 2741045 & $\mathrm{C}>\mathrm{T}$ & - & - & 0.007 & 1.000 \\
\hline M04 & UGT1A9 promoter & rs2741046 & $\mathrm{T}>\mathrm{A}$ & - & - & 0.007 & 1.000 \\
\hline M05 & UGT1A9 promoter & rs3832043 & $\mathrm{T} 9>\mathrm{T} 10$ & $* 1 \mathrm{~b}$ & - & 0.616 & 0.818 \\
\hline M06 & UGT1A9 promoter & - & $\mathrm{C}>\mathrm{T}$ & - & - & 0.007 & 1.000 \\
\hline M07 & UGT1A9 exon 1 & rs151216459 & $\mathrm{G}>\mathrm{T}$ & - & Synonymous & 0.014 & 1.000 \\
\hline M08 & UGT1A7 promoter & rs4530361 & $\mathrm{A}>\mathrm{G}$ & - & - & 0.250 & 0.633 \\
\hline M09 & UGT1A7 promoter & - & $\mathrm{T}>\mathrm{C}$ & - & - & 0.007 & 1.000 \\
\hline M10 & UGT1A7 promoter & rs28946877 & $\mathrm{C}>\mathrm{T}$ & - & - & 0.143 & 1.000 \\
\hline M11 & UGT1A7 promoter & rs7586110 & $\mathrm{T}>\mathrm{G}$ & - & - & 0.246 & 0.296 \\
\hline M12 & UGT1A7 exon 1 & rs 7577677 & $\mathrm{C}>\mathrm{A}$ & - & Synonymous & 0.243 & 0.314 \\
\hline M13 & UGT1A7 exon 1 & rs 17868323 & $\mathrm{~T}>\mathrm{G}$ & *2\&*3 & N129K & 0.407 & 1.000 \\
\hline M14 & UGT1A7 exon 1 & rs 17863778 & $\mathrm{C}>\mathrm{A}$ & *2\&*3 & $\mathrm{R} 131 \mathrm{~K}$ & 0.407 & 1.000 \\
\hline M15 & UGT1A7 exon 1 & rs 17868324 & $\mathrm{G}>\mathrm{A}$ & *2\&*3 & R131Q & 0.407 & 1.000 \\
\hline M16 & UGT1A7 exon 1 & rs139969318 & $A>G$ & - & Synonymous & 0.007 & 1.000 \\
\hline M17 & UGT1A7 exon 1 & rs11692021 & $\mathrm{T}>\mathrm{C}$ & $* 3$ & W208R & 0.25 & 0.633 \\
\hline M18 & UGT1A7 exon 1 & rs45462096 & $\mathrm{C}>\mathrm{T}$ & - & Synonymous & 0.014 & 1.000 \\
\hline M19 & UGT1A7 exon 1 & rs 17864686 & $\mathrm{G}>\mathrm{A}$ & - & Synonymous & 0.157 & 1.000 \\
\hline M20 & UGT1A1 promoter & rs887829 & $\mathrm{C}>\mathrm{T}$ & *80 & - & 0.107 & 1.000 \\
\hline M21 & UGT1A1 promoter & rs873478 & $\mathrm{G}>\mathrm{C}$ & *81 & - & 0.036 & 1.000 \\
\hline M22 & UGT1A1 promoter & rs3064744 & TA6>TA7 & *28 & - & 0.093 & 1.000 \\
\hline M23 & UGT1A1 exon 1 & rs4148323 & $\mathrm{G}>\mathrm{A}$ & *6 & G71R & 0.225 & 0.550 \\
\hline
\end{tabular}

MAF, minor allele frequency; UGT1A, UDP glucuronosyltransferase family 1 member A complex locus; H-Wpval, Hardy-Weinberg P-value.

In total, 6 SNPs exhibited an association with grade 3-4 neutropenia, including M01 ( $\mathrm{P}=0.009), \mathrm{M} 08(\mathrm{P}=0.011)$, M10 ( $\mathrm{P}=0.025)$, 17 ( $\mathrm{P}=0.011), \mathrm{M} 19(\mathrm{P}=0.014)$ and M23 $(\mathrm{P}=0.016)$ (Table IV). In addition, a significant association was observed between the UGT1A9*1b polymorphism (M05) and severe diarrhea $(\mathrm{P}=0.045$; Table IV). Of these 7 SNPs, two are functional mutations, consisting of UGT1A7*3 $(\mathrm{P}=0.011$; OR, 3.391) and UGT1A1*6 (P=0.016; OR, 3.373). The hotspot allele UGT1A1*28, which has been previously reported (20), was not significantly associated with grade 3-4 neutropenia or severe diarrhea $(\mathrm{P}=1.000$ and 0.620 , respectively; Table IV). The present study established haplotypes of those 7 SNPs and calculated the association with irinotecan-induced toxicity (Table V). Of the 7 haplotypes that were assessed, haplotypes H03, H04, H05 and H06 were observed to be associated with a higher risk of grade 3-4 neutropenia $(\mathrm{P}=0.037, \mathrm{P}=0.043$, $\mathrm{P}=0.097$ and $\mathrm{P}=0.004$, respectively). In addition, $\mathrm{H} 04$ was found to be associated with grade 3-4 diarrhea $(\mathrm{P}=0.001)$.

Pairwise LD analysis was performed with the detected variations having a MAF $>10 \%$. LD blocks were defined by the 'solid spine of LD' algorithm, and LD structure was exhibited by the GOLD heatmap color scheme. As shown in Fig. 1, a linkage block was observed across the sequenced region in the present metastatic gastrointestinal cancer patients. The variants detected in the UGT1A7 and UGT1A9 regions were closely associated (block 1). Out of the 6 SNPs that exhibited an association with grade 3-4 neutropenia, 5 SNPs were located in the UGT1A7-UGT1A9

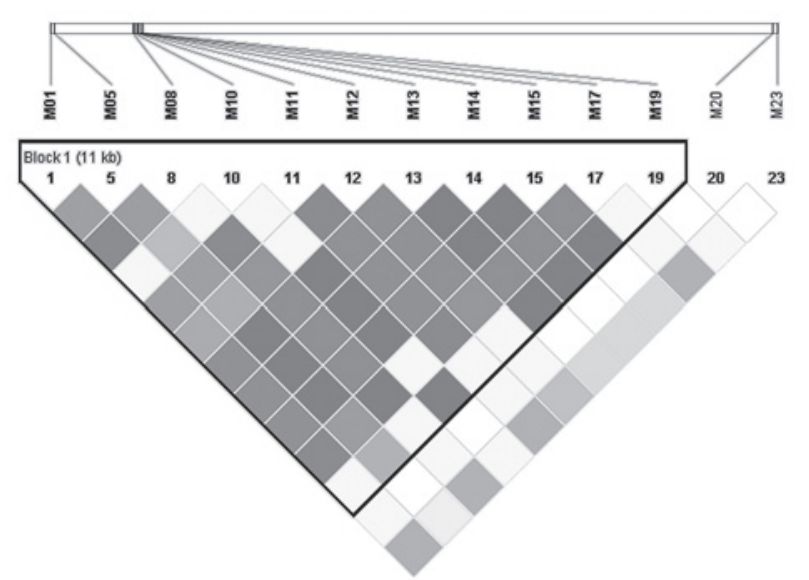

Figure 1. Pairwise linkage disequilibrium associations between UGT1A polymorphisms in metastatic gastrointestinal cancer patients. A denser color indicates greater linkage. An area corresponding to a haplotype block is exhibited in frame. UGT1A, UDP glucuronosyltransferase family 1 member A complex locus.

block (block 1). In addition, M17 (UGT1A7*3) was the only functional variant among the 5 SNPs. It was hypothesized that the significant association with grade 3-4 neutropenia of the other 4 SNPs was attributed to UGT1A7*3.

In total, 2 functional mutations exhibited a significant association with grade 3-4 neutropenia, including UGT1A7*3 
Table IV. Association between UGT1A polymorphisms and severe toxicity.

\begin{tabular}{|c|c|c|c|c|c|c|c|}
\hline \multirow[b]{2}{*}{ SNP ID } & \multirow[b]{2}{*}{ db SNP ID } & \multicolumn{3}{|c|}{ Neutropenia grade 3-4 } & \multicolumn{3}{|c|}{ Diarrhea grade 3-4 } \\
\hline & & P-value & OR & $95 \%$ CI & P-value & OR & $95 \% \mathrm{CI}$ \\
\hline M01 & rs3806598 & 0.009 & 3.584 & $1.457-8.821$ & 0.745 & 1.280 & $0.374-4.379$ \\
\hline M02 & rs45440791 & 1.000 & 0.000 & NA & 1.000 & 0.000 & NA \\
\hline M03 & rs 2741045 & 1.000 & 0.000 & NA & 0.100 & NA & NA \\
\hline M04 & rs 2741046 & 1.000 & 0.000 & NA & 0.100 & NA & NA \\
\hline M05 & rs3832043 & 1.000 & 1.003 & $0.417-2.411$ & 0.045 & 3.273 & $1.033-10.37$ \\
\hline M06 & - & 0.186 & NA & NA & 1.000 & 0.000 & NA \\
\hline M07 & rs 151216459 & 1.000 & 0.000 & NA & 1.000 & 0.000 & NA \\
\hline M08 & rs4530361 & 0.011 & 3.391 & $1.384-8.312$ & 0.339 & 1.778 & $0.553-5.714$ \\
\hline M09 & - & 1.000 & 0.000 & NA & 0.100 & NA & NA \\
\hline M10 & rs28946877 & 0.025 & 0.000 & NA & 1.000 & 1.000 & $0.206-4.845$ \\
\hline M11 & rs7586110 & 0.081 & 2.292 & $0.923-5.693$ & 1.000 & 0.818 & $0.214-3.124$ \\
\hline M12 & rs 7577677 & 0.077 & 2.344 & $0.944-5.819$ & 1.000 & 0.836 & $0.219-3.19$ \\
\hline M13 & rs17868323 & 0.659 & 1.314 & $0.558-3.099$ & 0.084 & 2.925 & $0.925-9.245$ \\
\hline M14 & rs66534818 & 0.659 & 1.314 & $0.558-3.099$ & 0.084 & 2.925 & $0.925-9.245$ \\
\hline M15 & rs17868324 & 0.659 & 1.314 & $0.558-3.099$ & 0.084 & 2.925 & $0.925-9.245$ \\
\hline M16 & rs139969318 & 0.186 & NA & NA & 1.000 & 0.000 & NA \\
\hline M17 & rs11692021 & 0.011 & 3.391 & $1.384-8.312$ & 0.339 & 1.778 & $0.553-5.714$ \\
\hline M18 & rs45462096 & 1.000 & 0.000 & NA & 1.000 & 0.000 & NA \\
\hline M19 & rs17864686 & 0.014 & 0.000 & NA & 0.236 & 2.400 & $0.679-8.481$ \\
\hline M20 & rs887829 & 0.737 & 0.647 & $0.137-3.062$ & 0.647 & 1.449 & $0.292-7.199$ \\
\hline M21 & rs873478 & 0.584 & 0.000 & NA & 0.414 & 2.346 & $0.244-22.59$ \\
\hline M22 & rs3064744 & 1.000 & 0.780 & $0.162-3.754$ & 0.620 & 1.742 & $0.345-8.802$ \\
\hline M23 & rs4148323 & 0.016 & 3.373 & $1.350-8.431$ & 0.191 & 0.241 & $0.03-1.92$ \\
\hline
\end{tabular}

SNP, single nucleotide polymorphism; OR, odds ratio; CI, confidence interval; UGT1A, UDP glucuronosyltransferase family 1 member A complex locus.

Table V. Correlation between UGT1A haplotypes and severe toxicity.

\begin{tabular}{|c|c|c|c|c|c|c|c|c|c|c|}
\hline \multirow[b]{2}{*}{ Haplotype ID } & \multicolumn{7}{|c|}{ UGT1A haplotype } & \multirow[b]{2}{*}{ Frequency } & \multicolumn{2}{|c|}{ P-value } \\
\hline & M01 & M05 & M08 & M10 & M17 & M19 & M23 & & Neutropenia grade 3-4 & Diarrhea grade 3-4 \\
\hline H01 & A & $10 \mathrm{~T}$ & A & $\mathrm{C}$ & $\mathrm{T}$ & G & G & 0.555 & 0.269 & 0.114 \\
\hline H02 & $\mathrm{C}$ & $9 \mathrm{~T}$ & G & $\mathrm{C}$ & $\mathrm{C}$ & G & A & 0.176 & 0.183 & 0.274 \\
\hline H03 & A & $9 \mathrm{~T}$ & A & $\mathrm{T}$ & $\mathrm{T}$ & $\mathrm{A}$ & G & 0.120 & 0.037 & 0.783 \\
\hline H04 & $\mathrm{C}$ & $9 \mathrm{~T}$ & $\mathrm{G}$ & $\mathrm{C}$ & $\mathrm{C}$ & G & G & 0.044 & 0.043 & 0.001 \\
\hline H05 & A & $10 \mathrm{~T}$ & A & $\mathrm{C}$ & $\mathrm{T}$ & G & A & 0.030 & 0.097 & 0.502 \\
\hline H06 & $\mathrm{C}$ & $10 \mathrm{~T}$ & G & $\mathrm{C}$ & $\mathrm{C}$ & G & A & 0.015 & 0.004 & 0.629 \\
\hline H07 & A & $9 \mathrm{~T}$ & $\mathrm{G}$ & $\mathrm{C}$ & $\mathrm{C}$ & $\mathrm{G}$ & $\mathrm{G}$ & 0.014 & 0.494 & 0.059 \\
\hline
\end{tabular}

UGT1A, UDP glucuronosyltransferase family 1 member A complex locus.

$(\mathrm{P}=0.011$; OR, 3.391; 95\% CI, 1.384-8.312) and UGT1A1*6 $(\mathrm{P}=0.016$; OR, 3.373; 95\% CI, 1.350-8.431). The risk of severe neutropenia associated with UGT1A7*3 and UGT1A1*6 genotypes (Table VI), and the combined effects of those two alleles, was analyzed (Table VII). In the present study, patients with one UGT1A7 3 allele had an OR of 4.444 (95\% CI, 1.060-18.627) for the risk of severe neutropenia.
Compared with patients heterozygous for UGT1A7*3, patients homozygous for UGT1A7*3 demonstrated a 5-fold increase in risk, with an OR of 23.333 (95\% CI, 1.609-338.419). The OR for patients homozygous for UGT1A1*6 (OR, 12.333; 95\% CI, 0.607-250.508) was two times higher than the OR of patients heterozygous for UGT1A1*6 (OR, 6.167; 95\% CI, 1.486-25.586). Combined effects analysis of those 2 alleles 
Table VI. Risk of severe toxicity associated with UGT1A7*3, UGT1A1*6 and UGT1A9*1b.

\begin{tabular}{|c|c|c|c|c|c|c|c|}
\hline SNP ID & Allele & Genotypes $^{\mathrm{a}}$ & Severe toxicity, $\mathrm{n}$ & Overall, $\mathrm{n}$ & P-value & OR & $95 \% \mathrm{CI}$ \\
\hline \multirow[t]{3}{*}{ M17 } & UGT1A7*3 & 0 & $3^{\mathrm{b}}$ & 38 & & & \\
\hline & & 1 & $8^{\mathrm{b}}$ & 29 & 0.046 & 4.444 & $1.060-18.627$ \\
\hline & & 2 & $2^{\mathrm{b}}$ & 3 & 0.035 & 23.333 & $1.609-338.419$ \\
\hline \multirow[t]{3}{*}{ M23 } & UGT1A1*6 & 0 & $3^{\mathrm{b}}$ & 40 & & & \\
\hline & & 1 & $9^{b}$ & 27 & 0.010 & 6.167 & $1.486-25.586$ \\
\hline & & 2 & $1^{\mathrm{b}}$ & 2 & 0.184 & 12.333 & $0.607-250.508$ \\
\hline \multirow[t]{3}{*}{ M05 } & UGT1A9*1b & 0 & $1^{\mathrm{c}}$ & 27 & & & \\
\hline & & 1 & $3^{\mathrm{c}}$ & 31 & 0.617 & 2.786 & $0.272-28.496$ \\
\hline & & 2 & $3^{c}$ & 11 & 0.065 & 9.750 & 0.886-107.249 \\
\hline
\end{tabular}

${ }^{a}$ Number of alleles carried by the patients. ${ }^{b}$ Number of patients developing neutropenia among all the patients in the same genotype group. ${ }^{\mathrm{c}}$ Number of patients developing diarrhea among all the patients in the same genotype group. SNP, single nucleotide polymorphism; OR, odds ratio; CI, confidence interval; UGT1A, UDP glucuronosyltransferase family 1 member A complex locus.

Table VII. Risk of severe neutropenia associated with combined effects of UGT1A7*3 and UGT1A1*6.

\begin{tabular}{lccccccc}
\hline SNP ID & Allele & Genotypes & Severe neutropenia, $n$ & Overall, $n$ & P-value & OR & 95\% CI \\
\hline M17 & UGT1A7 $3^{*}$ & 0 & $3^{\mathrm{a}}$ & $38^{\mathrm{b}}$ & 0.014 & 5.556 & $1.371-22.507$ \\
& & $1+2$ & $10^{\mathrm{a}}$ & $31^{\mathrm{b}}$ & & & \\
M23 & UGT1A1 $^{*} 6$ & 0 & $3^{\mathrm{a}}$ & $40^{\mathrm{b}}$ & 0.010 & 6.491 & $1.595-26.423$ \\
& & $1+2$ & $10^{\mathrm{a}}$ & $29^{\mathrm{b}}$ & & & \\
M17\&M23 & & 0 & $2^{\mathrm{a}}$ & $34^{\mathrm{b}}$ & 0.012 & 7.333 & $1.485-36.209$ \\
& & Others & $11^{\mathrm{a}}$ & $35^{\mathrm{b}}$ & & & \\
\hline
\end{tabular}

${ }^{a}$ Number of patients developing toxicity among all the patients in the same genotype group. ${ }^{b}$ Number of alleles carried by the patient. SNP, single nucleotide polymorphism; OR, odds ratio; CI, confidence interval; UGT1A, UDP glucuronosyltransferase family 1 member A complex locus.

revealed that patients carrying the UGT1A7*3 and UGT1A1*6 alleles had a higher OR (OR, 7.333; 95\% CI, 1.485-36.209) than the OR of patients carrying either UGT1A7*3 (OR, 5.556; 95\% CI; 1.371-22.507) or UGT1A1*6 (OR, 6.491; 95\% CI, 1.595-26.423). However, UGT1A9*1b was not significantly associated with the risk of severe toxicity in the heterozygous $(\mathrm{P}=0.617)$ or homozygous $(\mathrm{P}=0.065)$ state.

\section{Discussion}

Polymorphisms in the UGT1A family have been demonstrated to repeatedly affect the variability of irinotecan-induced toxicity, and the results are dependent on ethnicity. The toxicity of irinotecan remains unpredictable and requires additional investigation $(11,12,20)$.

Direct sequencing was adopted to avoid ethnic heterogeneity and to identify novel variations. Subsequent to sequencing of the promoter $(-1000 \mathrm{bp})$ and exon 1 regions of UGT1A1, UGT1A7 and UGT1A9, comprehensive analysis of genetic polymorphisms in these alleles was performed in Chinese patients with metastatic gastrointestinal cancer treated using irinotecan-based regimens. A total of 23 different genetic variants were detected, including 2 novel polymorphisms. In total, 7 SNPs exhibited an association with grade 3-4 toxicity
$(\mathrm{P}<0.05)$. Finally, 3 polymorphisms were focused on, 2 of which were the functional mutations UGT1A7*3 and UGT1A1* 6 that exhibited a significant association with grade 3-4 neutropenia (UGT1A7*3: $\mathrm{P}=0.011, \mathrm{OR}=3.391,95 \% \mathrm{CI}=1.384-8.312$; and UGT1A1*6: $\mathrm{P}=0.016, \mathrm{OR}=3.373,95 \% \mathrm{CI}=1.350-8.431$ ). Patients that carried the UGT1A7* 3 and UGT1A $1 * 6$ alleles had an increased risk of grade 3-4 neutropenia (OR, 7.333; 95\% CI, 1.485-36.209). Another key mutation is UGT1A9*1b $(-118$ 9T $>10 \mathrm{~T})$, which is found predominantly in the Asian population, and leads to increased enzyme expression and glucuronidation rates $(16,19)$. This was the only mutation significantly associated with grade 3-4 diarrhea $(\mathrm{P}=0.045$; OR, 3.273; 95\% CI, 1.033-10.37) in the present study.

UGT1A7*3 is located on UGT1A7 exon 1, and a transition of $\mathrm{T}>\mathrm{C}$ results in the 208th amino acid, tryptophan, changing to arginine. The UGT1A7*3 allele has been reported to generate an enzyme with $50 \%$ reduced catalytic activity (27). It has also been reported that $\mathrm{UGT} 1 \mathrm{~A} 7 * 3$ affects the pharmacokinetics of SN-38 in studies investigating both Caucasian and Asian patients $(11,16,18,20)$. In the present study, patients homozygous for UGT1A7*3 exhibited a 23.333-fold increased risk of grade 3-4 neutropenia (OR, 23.333; 95\% CI, 1.609-338.419), while patients heterozygous for UGT1A7*3 had an OR of 4.444 (95\% CI, 1.060-18.627). 
UGT1A $1^{*} 6$, a unique allele in the Asian population, is located on UGT1A1 exon 1 (11,12). A transition of $\mathrm{G}>\mathrm{A}$ results in a change of the 71st amino acid from glycine to arginine. Previous studies in the Asian population found that UGT1A $1^{*} 28 / /^{*} 6$ is a risk factor for irinotecan-induced toxicity $(18,28)$. The OR for UGT1A1*6-homozygous patients (OR, 12.333; 95\% CI, 0.607-250.508) was increased two-fold compared with the OR for UGT1A1*6-heterozygous patients (OR, 6.167; 95\% CI, 1.486-25.586).

In conclusion, the UGT1A1*6, UGT1A7*3 and UGT1A9*1b polymorphisms are predictive markers for severe toxicity in Chinese metastatic gastrointestinal cancer patients treated with irinotecan-based regimens.

\section{Acknowledgements}

The present study was supported by the National Natural Science Foundation of China (grant no. 81402999).

\section{References}

1. Mathijssen RH, van Alphen RJ, Verweij J, Loos WJ, Nooter K, Stoter G and Sparreboom A: Clinical pharmacokinetics and metabolism of irinotecan (CPT-11). Clin Cancer Res 7: 2182-2194, 2001

2. Guillemette C: Pharmacogenomics of human UDP-glucuronosyltransferase enzymes. Pharmacogenomics J 3: 136-158, 2003.

3. Cunningham D, Pyrhönen S, James RD, Punt CJ, Hickish TF, Heikkila R, Johannesen TB, Starkhammar H, Topham CA, Awad L, et al: Randomized trial of irinotecan plus supportive care versus supportive care alone after fluorouracil failure for patients with metastatic colorectal cancer. Lancet 352:1413-1418, 1998.

4. Saltz LB, Cox JV, Blanke C, Rosen LS, Fehrenbacher L, Moore MJ, Maroun JA, Ackland SP, Locker PK, Pirotta N, et al: Irinotecan plus fluorouracil and leucovorin for metastatic colorectal cancer. N Engl J Med 343: 905-914, 2000.

5. Fuchs CS, Moore MR, Harker G, Villa L, Rinaldi D and Hecht JR: Phase III comparison of two irinotecan dosing regimens in second-line therapy of metastatic colorectal cancer. J Clin Oncol 21: 807-814, 2003.

6. Zhang W, Zhao ZY, Wu Q, Li J, Fu Q, Cheng J, Xu N, Wu CP and Xu LG: Multicenter phase II study of modified FOLFIRI in patients in a Chinese population with advanced colorectal cancer (CRC) refractory to fluoropyrimidine and oxaliplatin. J Clin Oncol 23: 292s-292s, 2005.

7. Maitland ML, Grimsley C, Kuttab-Boulos H, Witonsky D, Kasza KE, Yang L, Roe BA and Di Rienzo A: Comparative genomics analysis of human sequence variation in the UGT1A gene cluster. Pharmacogenomics J 6: 52-62, 2006.

8. Gong QH, Cho JW, Huang T, Potter C, Gholami N, Basu NK, Kubota S, Carvalho S, Pennington MW, Owens IS and Popescu NC: Thirteen UDPglucuronosyltransferase genes are encoded at the human UGT1 gene complex locus. Pharmacogenetics 11: 357-368, 2001.

9. Iyer L, King CD, Whitington PF, Green MD, Roy SK, Tephly TR, Coffman BL and Ratain MJ: Genetic predisposition to the metabolism of irinotecan (CPT-11). Role of uridine diphosphate glucuronosyltransferase isoform 1A1 in the glucuronidation of its active metabolite (SN-38) in human liver microsomes. J Clin Invest 101: 847-854, 1998.

10. Gagné JF, Montminy V, Belanger P, Journault K, Gaucher G and Guillemette C: Common human UGT1A polymorphisms and the altered metabolism of irinotecan active metabolite 7-ethyl-10-hydroxycamptothecin (SN-38). Mol Pharmacol 62: 608-617, 2002.

11. Han JY, Lim HS, Shin ES, Yoo YK, Park YH, Lee JE, Jang IJ, Lee DH and Lee JS: Comprehensive analysis of UGT1A polymorphisms predictive for pharmacokinetics and treatment outcome in patients with non-small-cell lung cancer treated with irinotecan and cisplatin. J Clin Oncol 24: 2237-2244, 2006.
12. Minami H, Sai K, Saeki M, Saito Y, Ozawa S, Suzuki K, Kaniwa N, Sawada J, Hamaguchi T, Yamamoto N, et al: Irinotecan pharmacokinetics/pharmacodynamics and UGT1A genetic polymorphisms in Japanese: Roles of UGT1A1" 6 and " 28 . Pharmacogenet Genomics 17: 497-504, 2007.

13. Pharmacia and Upjohn Company LLC: Camptosar - irinotecan hydrochloride injection, solution. http://labeling.pfizer.com/Show Labeling.aspx?id=533.

14. Sai K, Saeki M, Saito Y, Ozawa S, Katori N, Jinno H, Hasegawa R, Kaniwa N, Sawada J, Komamura K, et al: UGT1A1 haplotypes associated with reduced glucuronidation and increased serum bilirubin in irinotecan-administered Japanese patients with cancer. Clin Pharmacol Ther 75: 501-515, 2004.

15. Bowcock AM, Anderson LA, Friedman LS, Black DM, Osborne-Lawrence S, Rowell SE, Hall JM, Solomon E and King MC: THRA1 and D17S183 flank an interval of $<4 \mathrm{cM}$ for the breast-ovarian cancer gene (BRCA1) on chromosome $17 \mathrm{q} 21$. Am J Hum Genet 52: 718-722, 1993.

16. Fujita K, Ando Y, Nagashima F, Yamamoto W, Eodo H, Araki K, Kodama K, Miya T, Narabayashi M and Sasaki Y: Genetic linkage of UGT1A7 and UGT1A9 polymorphisms to UGT1A $1{ }^{*} 6$ is associated with reduced activity for SN-38 in Japanese patients with cancer. Cancer Chemother Pharmacol 60: 515-522, 2007.

17. Lankisch TO, Schulz C, Zwingers T, Erichsen TJ, Manns MP, Heinemann V and Strassburg CP: Gilbert's syndrome and irinotecan toxicity: Combination with UDP-glucuronosyltransferase 1A7 variants increases. Cancer Epidemiol Biomarkers Prev 17: 695-701, 2008.

18. Hazama S, Mishima H, Tsunedomi R, Okuyama Y, Kato T, Takahashi K, Nozawa H, Ando H, Kobayashi M, Takemoto $\mathrm{H}$, et al: $\mathrm{UGT}_{1 \mathrm{~A}} 1^{*} 6,1 \mathrm{~A} 7^{*} 3$, and $1 \mathrm{~A} 9^{*} 22$ genotypes predict severe neutropenia in FOLFIRI-treated metastatic colorectal cancer in two prospective studies in Japan. Cancer Sci 104: 1662-1669, 2013.

19. Yamanaka H, Nakajima M, Katoh M, Hara Y, Tachibana O, Yamashita J, McLeod HL and Yokoi T: A novel polymorphism in the promoter region of human UGT1A9 gene (UGT1A9*22) and its effects on the transcriptional activity. Pharmacogenetics 14: 329-332, 2004.

20. Cecchin E, Innocenti F, D'Andrea M, Corona G, De Mattia E, Biason P, Buonadonna A and Toffoli G: Predictive role of the UGT1A1, UGT1A7, and UGT1A9 genetic variants and their haplotypes on the outcome of metastatic colorectal cancer patients treated with fluorouracil, leucovorin, and irinotecan. J Clin Oncol 27: 2457-2465, 2009.

21. Cui C, Shu C, Yang Y, Liu J, Shi S, Shao Z, Wang N, Yang T and $\mathrm{Hu} \mathrm{S}$ : XELIRI compared with FOLFIRI as a second-line treatment in patients with metastatic colorectal cancer. Oncol Lett 8: 1864-1872, 2014

22. Barrett JC, Fry B, Maller J and Daly MJ: Haploview: Analysis and visualization of LD and haplotype maps. Bioinformatics 21 : 263-265, 2005.

23. Barrett JC, Fry B, Maller J and Daly MJ: Haploview: Analysis and visualization of LD and haplotype maps. Bioinformatics 21: 263-265, 2005.

24. Abecasis GR and Cookson WO: GOLD-graphical overview of linkage disequilibrium. Bioinformatics 16: 182-183, 2000.

25. Uhlén M, Fagerberg L, Hallström BM, Lindskog C, Oksvold P, Mardinoglu A, Sivertsson Å, Kampf C, Sjöstedt E, Asplund A, et al: Proteomics. Tissue-based map of the human proteome. Science 347: 1260419, 2015.

26. Purcell S, Neale B, Todd-Brown K, Thomas L, Ferreira MAR, Bender D, Maller J, Sklar P, de Bakker PIW, Daly MJ and Sham PC: PLINK: A toolset for whole-genome association and population-based linkage analysis. Am J Hum Genet 81: 559-575.

27. Guillemette C, Ritter JK, Auyeung DJ, Kessler FK and Housman DE: Structural heterogeneity at the UDP-glucuronosyltransferase 1 locus: Functional consequences of three novel missense mutations in the human UGT1A7 gene. Pharmacogenetics 10: 629-644, 2000.

28. Wang H, Bian T, Jin T, Chen Y, Lin A and Chen C: Association analysis of UGT1A genotype and haplotype with SN-38 glucuronidation in human livers. Pharmacogenomics 15: 785-798, 2014. 